\title{
Droit au double degré de juridiction comme garantie du droit à un procès équitable : cas de la Cour militaire opérationnelle dans l'affaire Minova.
}

\author{
Par Mushekuru Mugeni Joyeux*
}

\section{Introduction}

Les juridictions militaires en RDC étaient, avant la loi de 2013 sur l'organisation, le fonctionnement et la compétence des juridictions de l'ordre judiciaire les seules juridictions à devoir réprimer les crimes internationaux. L'affaire ${ }^{1}$ qui fonde cette présente réflexion est celle jugée par la Cour militaire opérationnelle (CMO) du Nord Kivu en rapport avec les crimes $^{2}$ commis par les forces armées de la République démocratique du Congo (FARDC) dans la localité de Minova ${ }^{3}$ au Sud Kivu ${ }^{4}$. Plusieurs questions de droit s'étaient posées devant cette Cour. Mais, celle qui nous intéresse se rapporte au respect du droit à un procès équitable devant une juridiction qui statue en premier et dernier ressort.

En effet, cette juridiction a de particulier le fait que, pour une juridiction qui n'est pas l'une de plus hautes juridictions ${ }^{5}$, les voies de recours n'y soient pas consacrées. En effet, elle statue en premier et dernier ressort ${ }^{6}$. En plus, selon les termes de l'article 18 al. 2 du code judiciaire militaire, il s'agit d'une juridiction qui est implantée par le Président de la République ${ }^{7}$.

* Assistant à l'Université Catholique de Bukavu, Faculté de Droit. Contact mushekuru.mugeni@ucbukavu.ac.cd

1 Ce terme va désigner les arrêts rendus par la Cour militaire opérationnelle du Nord Kivu sous le RP 003.

2 Voy. Human Rights Watch, la Justice en procès, https://www.hrw.org/fr/report/2015/10/01/la-justice -en-proces/enseignements-tires-de-laffaire-des-viols-de-minova-en accédé dernièrement le 17 décembre 2017.

3 Localité au Nord de la Province du Sud Kivu en territoire de Kalehe.

4 Province située dans l'Est de la République Démocratique du Congo ayant pour chef-lieu la ville de Bukavu.

5 Dans la hiérarchie des juridictions congolaise, l'on trouve au sommet la tripartite Cour constitutionnelle, cour de Cassation et le Conseil d'Etat. Voy. Articles 153, 154 et 156 de la Constitution telle que modifiée par la Loi $\mathrm{n}^{\circ} 11 / 002$ du 20 janvier 2011 portant révision de certains articles de la Constitution de la République Démocratique du Congo du 18 février 2006, ». Journal officiel de la République démocratique du Congo, $52^{2 \grave{m e}}$ année, Numéro Spécial du 5 février 2011.

6 L'article 87 la loi ${ }^{\circ} 023 / 2002$ du 18 novembre 2002 portant code judiciaire militaire dispose que «Les arrêts rendus par les Cours Militaires Opérationnelles ne sont susceptibles d'aucun recours ». Journal Officiel, Numéro Spécial du 20 mars 2003.p. 19.

7 C'est dans ce sens que la Cour opérationnelle militaire du Nord Kivu a été implantée par l'Ordonnance $\mathrm{n}^{\circ} 08 / 003$ du 09 janvier 2008 portant implantation d'une Cour Militaire Opérationnelle. Journal officiel de la République démocratique du Congo $49^{\text {ème }}$ année, $\mathrm{N}^{\circ} 2$ du 15 janvier 2008. 
Ainsi la Cour militaire opérationnelle du Nord Kivu est en conformité avec les prescrits de la loi de 2002 portant code judiciaire militaire. Par contre, si la légalité de cette juridiction ne pose pas problème, l'on ne pourrait en dire autant de la conformité de cette loi à la nouvelle Constitution de la RDC telle que modifiée par la Loi n ${ }^{\circ} 11 / 002$ du 20 janvier 2011 portant révision de certains articles de la Constitution de la République Démocratique du Congo du 18 février 2006. Effet, cette dernière pose les principes garantissant aux justiciables le droit à un procès équitable. Il s'agit d'un ensemble de garanties procédurales que doivent observer les juridictions pour prétendre aboutir à une décision juste, exempte d'iniquité à l'encontre des justiciables qu'ils soient prévenus ou parties civiles. L'une des garanties procédurales qui se rattache aux droits à un procès équitable est le droit de faire examiner sa cause par un second juge ${ }^{8}$.

La Constitution de la RDC consacre, à son article 21, le droit de tous de former un recours contre un jugement. Par ailleurs, le même article précise, in fine, que son exercice sera précisé par la loi. En effet, les lois ${ }^{9}$ traitant de la procédure devant les juridictions règlent son mode d'exercice. Il arrive que ce droit soit limité voire proscrite dans certains cas ${ }^{10}$.

En plus de la non-conformité à la Constitution, les parties civiles ont soutenu, dans cette même affaire, que l'exclusion des voies de recours est une violation des traités internationaux dûment ratifiés par la R.D. Congo. Effet, ceux se rapportant aux droits civils soulignent les droits de recours et plus précisément celui de faire examiner sa décision par une juridiction d'appel comme garantie à un procès équitable ${ }^{11}$.

Au regard de l'activité judiciaire de cette Cour Militaire dans la Province du Nord Kivu et de son éventuelle implantation dans d'autres provinces, il demeure opportun de s'interroger sur sa conformité à la Constitution et aux instruments juridiques internationaux. C'est dans ce sens qu'il y a lieu de poser la question ci-après : l'exclusion des voies de recours devant la Cour militaire opérationnelle, telle qu'organisée par le code militaire congolais, est-elle une violation des droits à un procès équitable? Les parties civiles peuvent-elles s'en prévaloir et dans ce cas devant quelle instance?

8 Voy. Serge BRAUDO, Dictionnaire de droit privé, https://www.dictionnaire-juridique.com/definiti on/proces-equitable.php; Julie MEUNIER, « La notion de procès équitable devant la Cour européenne des droits de l'homme »,https://halshs.archivesouvertes.fr/halshs00419087/file/La_notion de_proces_equitable_devant_la_Cour_europeenne_des_droits_de_1_homme_-_MEUNIER_Julie.p df; Abderrachid Abdessemed «Principe du double degré de juridiction et les juridictions pénales internationaux » in Revue trimestrielle de Droits de l'Homme (74/2008), Directives et principes sur le droit à un procès équitable et à l'assistance judiciaire en Afrique, http://www.achpr.org/files/inst ruments/principles-guidelines-right-fair trial/achpr33_guide_fair_trial_legal_assistance_2003_fra.pdf; accédé dernièrement le 1 novembre 2016.

9 Lire la Loi organique $n^{\circ} 13 / 011-\mathrm{B}$ du 11 avril 2013 portant organisation, fonctionnement et compétences des juridictions de l'ordre judiciaire.

10 Les privilégiés de juridictions sont limités dans l'exercice de ce droit. C'est le cas des ceux qui sont justiciables devant la Cour de cassation (art. 153 de la Constitution) ou la Cour constitutionnel (art. 163 de la Constitution).

11 Voir Article 14 du Pacte international relatif aux droits civils et politiques disponible au http://ww w.ohchr.org/FR/ProfessionalInterest/Pages/CCPR.aspx, accédé dernièrement le 21 octobre 2016. 
Pour répondre à ces questions, il est important de partir des instruments internationaux et internes d'application en RDC qui posent ce droit. Cela nous permettra d'identifier sa portée, ses titulaires et sa nature juridique.

Ainsi, nous comptons interpréter dans une neutralité axiologique le sens des normes se rapportant au droit qu'ont les justiciables au double degré de juridiction. Par ailleurs, nous tâcherons d'offrir une clarification conceptuelle qui se dégage de la jurisprudence des organes internationaux de protection des droits de l'homme et du droit congolais sur la notion de droit au second juge comme une consécration du droit à un procès équitable.

Pour répondre à cette question il s'agira d'analyser dans un premier temps le droit au second juge comme garantie d'un procès équitable dans les textes internationaux (A). Et dans un deuxième temps, il faudra, avant de dire si le juge dans l'affaire Minova a bien dit le droit, savoir si cette Cour, créée par une loi de 2002, est toujours conforme à la Constitution de 2006 (B).

\section{A. Droit au deuxième juge comme respect d'un procès équitable en droit international de droits de l'Homme}

Nous définissons les principes de droit à un procès équitable comme un ensemble des droits processuels offerts aux parties pour s'assurer que leur cause a été dûment examinée, offrant du coup plus de légitimité à la décision rendue.

Parmi les principaux textes internationaux en vigueur en RDC qui font référence au droit à un procès équitable, figurent le Pacte international relatif aux droits civils et politiques et la Charte africaine des droits de l'homme et des peuples. Ces deux textes nous intéressent principalement puisqu'ils ont instauré des mécanismes de contrôle effectifs du respect des droits qu'ils prônent. Nous partirons donc de ces instruments internationaux pour ensuite préciser à travers leur jurisprudence, la charge sémantique que porte ce droit au double degré de juridiction comme garantie d'un procès équitable. En effet, si ces textes posent ce droit, il n'en détermine pas le contour. C'est donc dans les décisions judiciaires qu'il faille chercher le contenu qu'il porte.

Le Pacte international relatif aux droits civils et politiques (PIDCP) dispose sur les droits à un procès équitable à son article 14. Quant au droit au double degré de juridiction, il est énoncé à l'alinéa 5 de ce même article comme un droit reconnu aux personnes déclarées coupables d'une infraction. Par ailleurs le Pacte reste muet au sujet de la victime ou des parties civiles.

Quant à l'article 7 qui évoque les garanties procédurales liées aux droits à un procès équitable dans la Charte africaine de droit de l'homme et des peuples, il ne fait mention du droit au double degré de juridiction. Cependant, Il serait déduit des dispositions de cet article qui affirme : « le droit de saisir les juridictions nationales compétentes de tout acte violant les droits fondamentaux qui lui sont reconnus et garantis par les conventions, les lois, règlements et coutumes en vigueur (...) ». Comme nous le verrons, il s'agit d'une interpré- 
tation qui ne se prête pas à l'évidence justifiant ainsi la Commission africaine à adopter la Résolution de Tunis.

Aucun de ces textes ne définit la notion de droit à un procès équitable. Les dispositions légales ne font que donner les différents droits qui le composent sens en déterminer le contour exact.

Pour Paul TAVERNIER « le droit à un procès équitable est un droit complexe et multiple, il est essentiellement un droit de nature processuelle, bien qu'il touche également au fond à certains égards (...) mais en réalité les exigences multiples du droit à un procès équitables convergent toutes vers un but unique : l'équité, ce qui souligne le caractère substantiel du droit par-delà son caractère éminemment procédural $»^{12}$.

Le concept d'équité serait donc au centre de tous ces droits. En effet, l'appareil judiciaire concours par son œuvre à restaurer ou établir ce qui est " juste », " équitable » entre toutes les parties voire dans ou pour la société.

\section{Le pacte international relatif aux droits civils et politiques}

Le Pacte ainsi que son Protocole facultatif ont été ratifiés par la RDC le 1 er novembre $1976^{13}$. De ce fait, ils peuvent être invoqués par les parties au procès devant les juridictions nationales puisque faisant partie de l'arsenal juridique congolais en vertu de l'article 153 de la Constitution qui dispose que : « Les Cours et Tribunaux, civils et militaires, appliquent les traités internationaux dûment ratifiés (...)».

Mais également, les justiciables qui épuiseraient les voies de recours internes seraient en droit de porter leur cause devant le Comité des droits de 1 'homme ${ }^{14}$, organe de surveillances des droits consacrés par le Pacte. Celui-ci connaîtrait les litiges qui lui sont soumis en vertu de l'article $1^{\text {er }}$ du Protocole facultatif suscité ${ }^{15}$. C'est au regard de cette compétence que lui reconnait la RDC que les décisions et les observations du Comité peuvent être commentées pour mieux comprendre comment cet organe décline le droit au second juge comme droit à un procès équitable.

12 Paul TARVENIER, «La protection des droits de l'homme par le Comité des droits de l'homme des Nations Unies. Les communications individuelles » dans Revue trimestrielle des droits de l'homme (1996). p.6. http://www.rtdh.eu/pdf/19963.pdf accédé dernièrement le 15 décembre 2016.

13 Voy. Nations Unies, https://treaties.un.org/Pages/ViewDetails.aspx?src=TREATY\&mtdsg_no=IV$5 \&$ chapter $=4 \&$ clang $=$ _fr accédé dernièrement le 05 décembre 2016.

14 Voy. Haut-Commissariat aux Droit de l'Homme, http://www.ohchr.org/FR/HRBodies/CCPR/Page s/CCPRIndex.aspx accédé dernièrement le 22 décembre 2016.

15 Ce protocole dispose à son article 1 : « Tout Etat partie au Pacte qui devient partie au présent Protocole reconnaît que le Comité a compétence pour recevoir et examiner des communications émanant de particuliers relevant de sa juridiction qui prétendent être victimes d'une violation, par cet Etat partie, de l'un quelconque des droits énoncés dans le Pacte. Le Comité ne reçoit aucune communication intéressant un Etat partie au Pacte qui n'est pas partie au présent Protocole ». 
Dans le Pacte, comme dit plus haut, c'est l'article 14 qui dispose sur les garanties procédurales. Ils sont posés en ces termes : « Tous [les hommes] sont égaux devant les tribunaux et les cours de justice. Toute personne a droit à ce que sa cause soit entendue équitablement (...) ». L'alinéa 5 du même article dispose que : « Toute personne déclarée coupable d'une infraction a le droit de faire examiner par une juridiction supérieure la déclaration de culpabilité et la condamnation, conformément à la loi ». Ce qui pose sans ambiguïté le droit à interjeter appel contre les décisions judiciaires. Tel que présenté, ce droit semble être reconnu uniquement à la personne condamnée c'est-à-dire les personnes contre lesquelles une peine a été prononcée.

Le Comité des droits de l'homme a déjà eu à rendre des décisions dans des affaires où le droit à un procès équitable avaient été soulevé par des particuliers dans leurs communications et plus précisément sur la question de l'examen du juge supérieur.

Le Comité défend une lecture globale des paragraphes qui forment l'article 14. En effet, pour le Comité, il ne suffit donc pas que la possibilité du juge supérieur soit prévue mais aussi que la décision du premier juge, par le fait qu'elle soit écrite et motivée, soit un moyen permettant sa saisine. Ainsi, pour que le droit de double juridiction soit exercé par le condamné, il faut au préalable que dans un temps raisonnable une décision écrite lui soit disponible. Et donc, le Comité trouve un lien entre le droit d'être jugé sans retard excessif (Article 14. 3. C. du Pacte) et le droit au double degré de juridiction (Article 14.5. du Pacte). On peut affirmer que la méthode d'interprétation du Comité se trouve être téléologique $^{16}$. Il permet incontestablement de voir un lien entre les différents droits consacrés par l'article $14 \mathrm{du}$ PIDCP se rapportant tous aux garanties procédurales du droit à un procès équitable.

C'est ce qui ressort dans l'affaire Currie c/ la Jamaïque ${ }^{17}$.Dans cette affaire, le Comité demande que l'article 14 soit «In capital punishment cases, the obligation of States parties to observe rigorously all the guarantees for a fair trial set out in article 14 of the Covenant admits of no exception. The failure to provide Mr. Currie ineffective right to appeal without undue delay in accordance with article 14, paragraphs 3 (c) and 5, of the Covenant, means

16 Ce qui reste conforme à l'art. 31 de la Convention de Vienne sur le droit de Traité qui dispose : "Un traité doit être interprété de bonne foi suivant le sens ordinaire à attribuer aux termes du traité dans leur contexte et à la lumière de son objet et de son but ».

17 « That article 14, paragraph 3 (c), and article 14, paragraph 5, are to be read together, so that the right to review of conviction and sentence must be made available without undue delay. In this connection, the Committee refers to its earlier jurisprudence and reaffirms that under article 14 , paragraph 5, a convicted person is entitled to have, within reasonable time, access to written judgments, duly reasoned, for all instances of appeal in order to enjoy the effective exercise of the right to have conviction and sentence reviewed by a higher tribunal according to law. The Committee is of the opinion that the failure of the Court of Appeal to issue a written judgment, 13 years after the dismissal of the appeal, constitutes a violation of article 14, paragraphs 3 (c) and 5» Comité des droits de l'homme des Nations Unies, Affaire Curie contre la Jamaïque, CCPR/C/44/D/377/1989 (decision on admissibility, dated 20 March 1992). 
that he did not receive a fair trial within the meaning of the Covenant. Consequently, he is entitled, under article 2, paragraph 3 (a), of the Covenant, to an effective remedy ${ }^{18}$.

Il s'agit d'une ouverture aux voies d'appel quasi obligatoire qui est consacrée dans cette affaire surtout lorsque le prévenu encourt une peine de mort. Pour le Rapport du Colloque de Montpellier l'on s'accorde à reconnaître que « cette décision très importante ne constitue pas, malgré les apparences, un revirement de jurisprudence. Elle est intervenue dans une affaire où il y avait eu condamnation à mort, ce qui a sans doute incité le Comité à examiner le cas avec une particulière attention et lui a permis de consacrer un véritable droit d'accès à un tribunal, y compris à un tribunal constitutionnel, quand il existe, ouvrant ainsi la voie à l'application de l'ensemble des droits et garanties prévus à l'article $14 \gg{ }^{19}$. Mais pour nous, il s'agit d'une affirmation gardant toute son importance. En effet, pour un texte ${ }^{20}$ qui n'interdit pas la peine de mort, il n'était pas question de priver au condamné une occasion de voir reformer sa décision de condamnation. Pour le Comité des droits de l'homme, le droit au second juge est obligatoire toute les fois que l'accusé encours une peine de mort.

Dans une toute autre affaire, Jésus TERRON c/ l'Espagne, le Comité des droits de l'homme affirme que les droits de réexamen du juge supérieur consacré par l'article 14 paragraphe 5 ne peut être violé même lorsqu'il s'agit des personnes bénéficiant du privilège de juridiction. Ainsi, elles ont droit que leurs causes soient de nouveau entendue quant à la déclaration de leur culpabilité et de leur condamnation. C'est ainsi que le Comité déclara : « Même si la législation de l'Etat partie dispose, en certaines circonstances, qu'en raison de sa charge une personne sera jugée par un tribunal de rang supérieur à celui qui serait normalement compétent, cette circonstance ne peut à elle seule porter atteinte au droit de l'accusé au réexamen de la déclaration de culpabilité et de la condamnation par un tribunal $»^{21}$. C'est ainsi que, le Comité conclut à la violation de l'article 14 paragraphe 5 du Pacte. Dans cette même affaire, il déclare également que la violation de l'article 14 paragraphe 5 justifie à elle seule qu'il y a eu épuisement des voies de recours interne et la violation du droit au recours utile dont fait mention l'article 2 paragraphe 3 . Cela est d'autant plus logique pour le Comité pour autant que le paragraphe 5 de l'article 14 du Pacte est « une lex specialis par rapport au paragraphe 3 a) de l'article $2(\ldots) »^{22}$.

18 Comité des droits de l'homme des Nations Unies, Affaire Curie contre la Jamaïque, CCPR/C/44/D/377/1989 (decision on admissibility, dated 20 March 1992).

19 TAVERNIER., Note 12. pp. 11-12.

20 Il s'agit du Pacte international relatif aux droits civils et politiques. En effet, ce texte ne proscrit pas la peine de mort. Pour s'en convaincre il suffit de lire l'article 6 aux alinéas 4 et 5 qui dispose respectivement : " Tout condamné à mort a le droit de solliciter la grâce ou la commutation de la peine. L'amnistie, la grâce ou la commutation de la peine de mort peuvent dans tous les cas être accordées ». " Une sentence de mort ne peut être imposée pour des crimes commis par des personnes âgées de moins de 18 ans et ne peut être exécutée contre des femmes enceintes ».

21 Comité des droits de l'homme des Nations unies, affaire Jésus TERRON cl Espagne, CCPR/C/82/D/1073/2002, 15 novembre 2004. p. 7.

22 Comité des droits de l'homme des Nations unies, affaire Jésus TERRON c/ Espagne, CCPR/C/82/D/1073/2002, 15 novembre 2004. p. 6. 
Plus encore, dans l'affaire M. Valichon Aliboev c/ le Tadjikistan, le Comité déclare, comme pour réaffirmer son interprétation que «l'absence de possibilité de faire appel devant une juridiction supérieure des jugements rendus par la Cour suprême en première instance ne satisfait pas aux prescriptions énoncées au paragraphe 5 de l'article 14 et qu'il y a donc eu violation de cette disposition $»^{23}$. Pour étayer son propos, le Comité cite sa jurisprudence pour préciser la constance qui caractérise ses décisions chaque fois qu'il y a violation de l'article 15 paragraphe 5 se rapportant au défaut du réexamen de la déclaration de culpabilité et de la condamnation par un Etat. Il s'agit entre autres : Khalilova c. Tadjikistan, communication n ${ }^{\circ}$ 973/2001, constatations adoptées le 30 mars 2005, Aliev c. Ukraine, communication $\mathrm{n}^{0} 781 / 1997$, constatations adoptées le 7 août 2003, Robinson c. Jamaïque, communication $\mathrm{n}^{\mathrm{0}}$ 223/1987, constatations adoptées le 30 mars 1989, Brown c. Jamaïque, communication $n^{\circ} 775 / 1997$, constatations adoptées le 23 mars $1999^{24}$.

S'il est vrai que le droit de double degré de juridiction est consacré par le Pacte, il reste cependant un droit dont le titulaire reste la personne déclarée coupable. Ce qui exclut de jure la partie civile. Il s'agit donc d'un droit indisponible pour les parties civiles. En effet, pour ester devant une instance judiciaire, il faut justifier d'un intérêt. Dans le cas du Comité, il doit s'agir d'une violation d'un droit garanti par le Pacte et dont on est reconnu titulaire ${ }^{25}$. Dans son observation générale $n^{\circ} 32$, le Comité des droits de l'homme précise sans ambiguïté que « Le paragraphe 5 de l'article 14 ne s'applique pas aux procédures portant sur des droits et obligations de caractère civil ni à aucune autre procédure qui n'est pas un élément du système d'appel pénal, comme les recours constitutionnels. (Communication no $352 / 1989$, Douglas, Gentles, Kerr c. Jamaïque, par. 11.2) ${ }^{26}$. Cependant, il ne dit rien en ce qui concerne les réparations - qui peuvent être des demandes à caractère civil dans un procès pénal - portées par la partie civile. L'on se demandera alors, comment les parties civiles, comme celle dans l'affaire Minova, pourraient évoquer la violation de ce droit au second juge devant le Comité des droits de l'homme?

Un tout autre raisonnement est dans ce cas possible pour justifier le droit au second juge à la partie civile. Le fait, pour la Cour militaire opérationnelle, de priver de voie de recours le condamné, en violation de l'al. 5 de l'article 14 du PIDCP a aussi une incidence directe sur le suite à donner à l'action civile des victimes. En effet, l'action en accusation est dans ce cas intimement liée à celle de la réparation. Cette dernière ne peut d'ailleurs

23 Comité des droits de l'homme des Nations unies, affaire M. Valichon Aliboev c/ le Tadjikistan, CCPR/C/85/D/985/2001. 16 novembre 2005. p. 8.

24 Ibidem.

25 «N'importe qui peut présenter une requête devant un comité contre un État qui satisfait à ces deux conditions [Premièrement, il doit être partie au traité en question, c'est-à-dire qu'il doit l'avoir ratifié ou accepté d'une autre manière (...) Deuxièmement, l'État partie doit avoir reconnu la compétence du comité créé par le traité pertinent pour examiner les requêtes individuelles], en disant que les droits qui lui sont reconnus par le traité ont été violés » disponible sur http://www.ohchr.org/D ocuments/Publications/FactSheet7Rev. 1 fr.pdf accédé dernièrement le 5 décembre 2016.

26 Idem. p. 18. 
aboutir qu'une fois l'infraction établie dans le chef de l'accusé. Ainsi, garantir un droit de recours au condamné et le priver à la partie victime mettraient en mal l'égalité ${ }^{27}$ entre les parties pourtant recherchées dans le principe d'équité défendu par le Pacte. Il faut rappeler que la compréhension de l'article 14 requiert une interprétation globale des droits établis.

Ainsi, si ce droit, tel qu'il est présenté est celui reconnu à l'accusé, l'on ne peut le concevoir sans les autres droits du même article qui décrivent la nature de l'instance judiciaire : compétent, indépendant, impartial et établi par la loi. Un tribunal qui dénie les droits d'appel ne saurait prétendre tout au moins à l'impartialité et cela à l'égard de toutes les parties au procès. Considérant que l'article 14 déclare l'égalité des tous devant les juridictions, l'accusé et la partie civile ne devraient pas dans ce cas être privé tous deux du droit de faire examiner leurs prétentions par une juridiction supérieure. L'un en ce qui concerne sa déclaration de culpabilité et de sa condamnation et l'autre quant à sa demande de réparation conformément aux principes fondamentaux de la Résolution ${ }^{28}$ 40/34 du 29 novembre 1985. Ainsi, grâce à la protection par ricochet, la victime ou partie civile recouvre également son droit à l'appel.

Si de par le droit international de droits de l'homme les garanties au double degré de juridiction pour les parties civiles restent discutables, le droit régional africain en ce domaine offre des atouts non moins négligeables.

\section{La charte africaine des droits de l'homme et des peuples}

Le droit de faire recours devant une juridiction supérieure n'est pas prévu dans la Charte africaine des droits de l'homme et des peuples. En effet, l'article 7 qui pose principalement les principes se rapportant à un procès équitable n'en fait pas mention expressis verbis ${ }^{29}$.

27 « En termes généraux, le droit à l'égalité devant les tribunaux et les cours de justice garantit, outre les principes mentionnés dans la deuxième phrase du paragraphe 1 de l'article 14, les principes de l'égalité d'accès et de légalité de moyens (Egalité des armes), et vise à ce que les parties à la procédure ne fassent l'objet d'aucune discrimination ».Comité des droits de l'homme, Observation générale $N^{\circ} 32$ Article 14. Droit à l'égalité devant les tribunaux et les cours de justice et à un procès équitable. Quatre-vingt-dixième session Genève, 9-27 juillet 2007. CCPR/C/GC/32 23 août 2007.p. 3. Disponible sur http://docstore.ohchr.org/SelfServices/FilesHandler.ashx?enc=6QkG1d \%2FPPRiCAqhKb7yhsrdB0H115979OVGGB\%2BWPAXhRj0XNTTvKgFHbxAcZSvX1Pm4Pby t3LF2duuUH3Ajd\%2FmxkulVu5jsenIKJixUXDrYVoLrDIBfi12Zp7jfRplT1 accédé dernièrement le 5 décembre 2016.

28 Déclaration des principes fondamentaux de justice relatifs aux victimes de la criminalité et aux victimes d'abus de pouvoir. Adoptée par l'Assemblée générale des Nations unies dans sa Résolution 40/34 du 29 novembre 1985.

29 Il dispose que : « Toute personne a droit à ce que sa cause soit entendue. Ce droit comprend: a. le droit de saisir les juridictions nationales compétentes de tout acte violant les droits fondamentaux qui lui sont reconnus et garantis par les conventions, les lois, règlements et coutumes en vigueur; b. le droit à la présomption d'innocence, jusqu'à ce que sa culpabilité soit établie par une juridiction compétente; c. le droit à la défense, y compris celui de se faire assister par un défenseur de son choix; d. le droit d'être jugé dans un délai raisonnable par une juridiction impartiale. 2. Nul ne peut être condamné pour une action ou une omission qui ne constituait pas, au moment où elle a eu lieu, 
Il serait plus difficile de déduire des autres dispositions de cette Charte l'exigence du réexamen par une juridiction supérieure. Cela est d'autant plus délicat puisque l'article 56 point 5 qui dispose sur l'une des conditions de la recevabilité des communications précise qu'elle doit : "Etre postérieures à l'épuisement des recours internes s'ils existent, (...) ». Ce qui pourrait s'interpréter comme étant une faculté donnée aux Etats d'avoir ou pas des instances de réexamen des décisions judiciaires.

Pour pallier cette lacune, la Commission africaine des droits de l'homme et des peuples, qui est l'organe de surveillance de la Charte ${ }^{30}$, a pris en 1992 une résolution ${ }^{31}$ sur la procédure relative au droit de recours et à un procès équitable.

Il s'agit de la Résolution dite de Tunis qui est une résolution thématique ${ }^{32}$ devant servir de base aux législations nationales dans la codification des règles se rapportant au droit à un procès équitable. Sans valeur contraignante ${ }^{33}$ pour les Etats parties, cette résolution joue par ailleurs un rôle dans l'interprétation que la Commission fait lors de l'examen des communications. C'est ainsi que cette Résolution pose le principe du droit au second juge au point 3 en ces termes : les "personnes accusées d'un délit auront le droit de faire appel devant une juridiction supérieure $»^{34}$.

Que l'absence de recours efficace, disponible et satisfaisant soit une condition de recevabilité d'une communication individuelle est une chose, par ailleurs une autre est de savoir s'il s'agit d'une violation d'un droit garanti?

Dans l'affaire Mamboleo c/ la RDC, la Commission après avoir défini la nature des voies de recours pour qu'elle les estime épuisées ${ }^{35}$, rejette les allégations de la RDC qui

une infraction légalement punissable. Aucune peine ne peut être infligée si elle n'a pas été prévue au moment où l'infraction a été commise. La peine est personnelle et ne peut frapper que le délinquant ». Disponible sur http://www.achpr.org/fr/instruments/achpr/ accédé dernièrement le 20 novembre 2016.

30 En vertu des articles 30,45 et suivant, article 55, 56, 57, 58 et 59 de la Charte africaine des droits de l'homme et des peuples.

31 En effet, en vertu de l'article 45 al. 1 ii) de la Charte africaine des droits de l'homme et des peuples, la Commission a pour mission de « formuler et élaborer, en vue de servir de base à l'adoption de textes législatifs par les gouvernements africains, des principes et règles qui permettent de résoudre les problèmes juridiques relatifs à la jouissance des droits de l'homme et des peuples et des libertés fondamentales $»$.

32 Voir http://www.achpr.org/fr/resolutions/about/ accédé dernièrement le 26 octobre 2016.

33 La commission africaine des droits de l'homme classe les résolutions dans la catégorie de la soft law. Ce qui implique qu'elles ne sont revêtues d'aucune force contraignante Voy. http://www.achpr .org/fr/instruments/ accédé dernièrement le 26 octobre 2016.

34 Résolution sur la Procédure relative au Droit de Recours et à un Procès Equitable adoptée par la Commission Africaine des Droits de l'Homme et des Peuples, réunie en sa 11ème Session Ordinaire, à Tunis Tunisie, du 2 au 9 mars 1992. Consulté sur http://www.achpr.org/fr/sessions/11th/res olutions/4/ accédé dernièrement le 22 octobre 2016.

35 « Elles doivent être « disponibles, adéquats et efficaces; 6 qu'un recours est disponible lorsque le plaignant peut l'introduire sans empêchement; qu'il est adéquat lorsqu'il est apte à redresser le tort; qu'il est efficace lorsqu'il offre des chances de succès » (d'où tires-tu la citation?). Ainsi en at-il décidé dans les affaires Jawara c. Gambie (2000) RADH 107 (2000) para 31; Liesbeth Zegveld 
affirmait que la cause portée par le requérant était encore pendante devant les juridictions congolaises en l'occurrence devant la section administrative de la Cour suprême de Justice. En effet, pour la Commission, "si l'affaire était effectivement pendant au moment de sa saisine, elle l'était devant une haute juridiction dont les décisions sont sans recours; que tout recours avait donc été épuisé, l'affaire étant déjà restée pendante devant la Cour suprême quatre ans durant à la date où le plaignant saisissait la Commission, soit le 20 avril $2005 »^{36}$. Pour la Commission, le prolongement abusif d'une cause devant une juridiction supérieure n'offrant pas de voie de recours possible est une justification de sa saisine puisque satisfaisant à l'exigence de l'épuisement des voies de recours interne disposé à l'article 56 al. 5.

Bref, il y a lieu de dire que pour la Commission, l'épuisement de voies de recours internes peut également se déduire lorsqu'une juridiction inférieure statue en premier et dernier ressort qu'importe la possibilité pour le plaignant d'aller en cassation puisqu'il s'agit là d'un recours peu efficace. Tel en a été décidé dans l'affaire Interights, ASADHO et Maître Odette Disu c/République Démocratique du Congo ${ }^{37}$.

Mais plus encore, cette affaire apporte un éclairage sur l'absence d'appel au sein des juridictions militaires congolaises. Avant de conclure de la violation du droit de faire appel par la RDC, la Commission y affirme que ce droit est garanti par le l'article 7 al. 1 a) de la Charte $^{38}$. Ainsi, elle déclare que : « La Commission dispose d'une jurisprudence abondante $^{39}$ sur le droit à l'appel, concernant en particulier des espèces impliquant les juridictions militaires et où la peine de mort était encourue. Le principe constant adopté est que les

et Mussie Ephrem c.Eritrea (2003) AHRLR 84 (ACHPR 2003), où peut-on trouver ces décisions et où les avais-tu trouvées? Voir aussi la jurisprudence de la Commission interaméricaine des droits de l'homme dans l'affaire Velasquez Rodriguez (29 juillet 1988) Série C №4 (1988). Jawara c. Gambie para 31; Article 19 c. Eritrea (2007) AHRLR 73 (ACHPR 2007) para 46.

36 Commission Africaine des droits de l'homme et des peuples, affaire Maître Mamboleo M. Itundamilamba c/ la République démocratique du Congo.achpr53_decis_302_05_drc_2013_fra. accédé dernièrement sur $\mathrm{http}: / / \mathrm{www}$.achpr.org/fr/communications $/ \mathrm{dec}-\overline{s i o n s} / \overline{\mathrm{c}}=\overline{67} \& \mathrm{a}=863$ accédé dernièrement le 25 octobre 2016.

37 La Commission a déclaré : « (...) En outre, en tant que juridiction de cassation, la Cour suprême ne constituait pas un recours efficace puisqu'elle n'aurait examiné que le respect des règles de procédure et non la substance de la cause. Par conséquent, elle n'était pas susceptible de réparer les violations alléguées ». Disponible sur http://www.achpr.org/fr/communications/decision/274.03_et _282.03_ accédé dernièrement le 5 décembre 2016.

38 Cet article qui dispose : «Toute personne a droit à ce que sa cause soit entendue. Ce droit comprend : a. le droit de saisir les juridictions nationales compétentes de tout acte violant les droits fondamentaux qui lui sont reconnus et garantis par les conventions, les lois, règlements et coutumes en vigueur; $(\ldots) »$.

39 Voy. Law Office of Ghazi Suleiman c. Soudan Communication 222/98 et 229/99 (2003) RADH 142

(CADHP 2003) para 53; Women's Legal Aid Centre (pour le compte de Moto) c. Tanzanie Communication 243/01 (2004) RADH 120 (CADHP 2004) para 47; Civil Liberties Organisations et autres c. Nigéria (2001) RADH 80 (CADHP 2001) para 32-34. 
tribunaux militaires ne bénéficient d'aucune dérogation quant au respect des droits liés au procès équitable. Le droit à l'appel, particulièrement en cas d'imposition de la peine de mort, est également consacré par la Commission dans ses Directives et principes sur le droit à un procès équitable $»^{40}$.Il s'agit d'un cas suffisamment similaire au cas Minova sous examen. En effet, comme la Cour militaire opérationnelle, la Cour d'ordre militaire rendait des décisions en premier et dernier ressort ${ }^{41}$. C'est d'ailleurs dans cette décision qu'il avait été demandé à la RDC de mettre ses lois en conformité avec la Charte ${ }^{42}$. Si des efforts en ce sens ont été reconnus par la Commission pour ce qui est de la loi ${ }^{\circ} 023$ du 18 novembre 2002 portant Code judiciaire militaire, elle souligne cependant « qu'une telle réforme ne règle que partiellement la non-conformité du droit pénal congolais avec les dispositions de la Charte africaine dont la violation a été constatée dans la présente Communication $»^{43}$. Cela est d'autant plus vrai que la disposition déniant tout droit au second juge a été maintenue pour ce qui est des décisions rendues par la Cour militaire opérationnelle ${ }^{44}$.

Mais alors, il est important de savoir si ce droit serait autant reconnu au prévenu qu'aux parties civiles devant la Commission puisque la résolution de Tunis parle des «Personnesaccusées $(\ldots) »$.

Pour ce qui concerne la Charte africaine des droits de l'homme et des peuples, le même raisonnement d'une protection par ricochet demeure valable. Plus encore, cet instrument, plus que le précédent, offre plus d'atout à son organe de surveillance. Il se rapporte au droit

40 Commission africaine des droits de l'homme et des peuples, affaire Interights, ASADHO et Maitre Odette Disu c/République Démocratique du Congo, Communication 274/03 et 282/03, p.14. Consulté sur http://www.achpr.org/fr/communications/decisions/?c=67\&a=863 accédé dernièrement le 22 octobre 2016.

41 Article 5 du Décret-loi no 019 du 23 août 1997 portant institution d'une Cour d'ordre militaire. Consulté sur http://www.achpr.org/files/sessions/44th/comunications/281.03/achpr44_281_03_fra. pdf. Accédé dernièrement le 5 décembre 2016.

42 Commission africaine des droits de l'homme et des peuples, affaire Interights, ASADHO et Maitre Odette Disu c/République Démocratique du Congo, Communication 274/03 et 282/03, p.20. Consulté sur http://www.achpr.org/fr/communications/decisions/?c=67\&a=863 le 22 octobre 2016. Veux-tu relever les paragraphes qui font cette demande à la RDC?

43 Idem, p.19. Consulté sur http://www.achpr.org/fr/communications $/$ decisions/?c=67\&a=863 le 22 octobre 2016.

44 Article 87 de la loi n⿳023/2002 du 18 novembre 2002portant code judiciaire militaire« Les arrêts rendus par les Cours Militaires Opérationnelles ne sont susceptibles d'aucun recours ».Journal Officiel - Numéro Spécial - 20 mars 2003.p. 19. 
applicable devant la Commission. En effet, les articles $60^{45}$ et $61^{46}$ de la Charte démontrent que la Commission dispose d'une palette d'instruments plus large pour l'interprétation de sa propre règle pendant la résolution des différends portés devant elle. C'est ainsi qu'elle en fait mention dans l'affaire Law office of Ghazi Suleiman c. Soudan lorsqu'en vertu de l'article 60, qui lui autorise à s'inspirer du droit international relatif au droit de l'homme, elle citera ainsi les jurisprudences de la Cour européenne de droits de l'homme et de la Commission interaméricaine des droits de l'homme ${ }^{47}$.

Pour Alain Didier OLINGA, cela conduit à un emprunt de triple nature de la Commission à ses homologues européenne et interaméricaine : des éléments de contenu substantiel des droits (emprunt substantiel), des éléments de référence du contrôle des ingérences dans l'exercice des droits et d'objectivation de certains comportements prohibés (emprunt méthodologique) et des éléments liés à la posture de l'organe de contrôle vis-à-vis des Etats ou relatifs à certains principes d'interprétation (emprunt interprétatif ${ }^{48}$.

Pour les victimes parties civiles dans l'affaire Minova, la commission africaine des droits de l'homme devient donc l'organe le mieux indiqué devant laquelle elles pourraient porter leur revendication.

De manière rassurante la RDC en 2006 s'est dotée d'une constitution qui affirme le droit à l'appel devant toute les juridictions. Ce qui implique qu'il y a lieu d'analyser la conformité de la loi de 2002 portant code judiciaire militaire à la loi fondamentale.

\section{B. De la constitutionnalité de la Cour militaire opérationnelle}

L'article 21 al. 2 dispose que : « Le droit de former un recours contre un jugement est garanti à tous. Il est exercé dans les conditions fixées par la loi ». Parlant des juridictions mili-

45 « La Commission s'inspire du droit international relatif aux droits de l'homme et des peuples, notamment des dispositions des divers instruments africains relatifs aux droits de l'homme et des peuples, des dispositions de la Charte des Nations Unies, de la Charte de l'Organisation de l'Unité Africaine, de la Déclaration Universelle des Droits de l'Homme, des dispositions des autres instruments adoptés par les Nations Unies et par les pays africains dans le domaine des droits de l'homme et des peuples ainsi que des dispositions de divers instruments adoptés au sein d'institutions spécialisées des Nations Unies dont sont membres les parties à la présente Charte ».

46 « La Commission prend aussi en considération, comme moyens auxiliaires de détermination des règles de droit, les autres conventions internationales, soit générales, soit spéciales, établissant des règles expressément reconnues par les Etats membres de l'Organisation de l'Unité Africaine, les pratiques africaines conformes aux normes internationales relatives aux droits de l'homme et des peuples, les coutumes généralement acceptées comme étant le droit, les principes généraux de droit reconnus par les nations africaines ainsi que la jurisprudence et la doctrine ».

47 Lire aussi l'affaire 255/98 Hurilaws c. Nigeria; l'affaire 236/2000 Curtis Doebbler c. Soudan; l'affaire 218/98 Civil liberties organization, Légal Defence Centre, Légal Defence and Assistance Project c. Nigeria; l'affaire 74/92, Commission nationale des droits de l'homme et des libertés c. Tchad.

48 Alain Didier OLINGA, « Les emprunts normatifs de la commission africaine des droits de l'homme et des peuples aux systèmes européen et interaméricain de garantie des droits de l'homme ». Consulté sur www.rtdh.eu/pdf/2005499.pdf. accédé dernièrement le 15 octobre 2016. 
taires, la Constitution donne pouvoir au Président de la République de suspendre l'action des cours et tribunaux civils au profit des juridictions militaire dans des cas bien précis : En temps de guerre ou lorsque l'état de siège ou d'urgence est proclamé. Cependant l'article 156 qui consacre cette compétence dispose à son al. 2 in fine que « (...) le droit d'appel ne peut être suspendu ». C'est en ces termes que le droit à une juridiction supérieure est énoncé dans la Constitution. Cette dernière pose sans ambiguïté le droit au double degré de juridiction en tout temps pour toutes les parties au procès.

Comme droit consacré par la Constitution, il s'impose à toutes les normes de valeur inférieure en vertu du principe de la hiérarchie des normes. Dans la Constitution, il est possible d'envisager, prenant en compte le moment où il s'exerce, deux sortes de contrôles législatifs : en amont (contrôle a priori) avant la promulgation des lois (art. 160) ${ }^{49}$ et en aval (contrôle a posteriori) en l'occurrence par la procédure de l'exception d'inconstitutionnalité évoquée par une partie au procès (art. 161 al. 3$)^{50}$.

Cependant, ce droit connaît des exceptions devant certaines juridictions. En effet, le recours contre les arrêts de hautes juridictions est impossible. C'est le cas des arrêts de la Cour constitutionnelle ${ }^{51}$. Même en ce qui concerne les privilégiés de juridiction ${ }^{52}$. Il en est de même pour la Cour de cassation et le Conseil d'Etat sauf dans l'hypothèse du déclinatoire de juridiction soulevé devant eux ${ }^{53}$. Par ailleurs signalons tout de même que ces ins-

49 Article 160 de la Constitution telle que modifiée par la Loi n ${ }^{\circ} 11 / 002$ du 20 janvier 2011 portant révision de certains articles de la Constitution de la République Démocratique du Congo du 18 février 2006 : « La Cour constitutionnelle est chargée du contrôle de la constitutionnalité des lois et des actes ayant force de loi. Les lois organiques, avant leur promulgation, et les Règlements Intérieurs des Chambres parlementaires et du Congrès, de la Commission électorale nationale indépendante ainsi que du Conseil supérieur de l'audiovisuel et de la communication, avant leur mise en application, doivent être soumis à la Cour constitutionnelle qui se prononce sur leur conformité à la Constitution. Aux mêmes fins d'examen de la constitutionnalité, les lois peuvent être déférées à la Cour constitutionnelle, avant leur promulgation, par le Président de la République, le Premier ministre, le Président de l'Assemblée nationale, le Président du Sénat ou le dixième des députés ou des sénateurs. La Cour constitutionnelle statue dans le délai de trente jours. Toutefois, à la demande du Gouvernement, s'il y a urgence, ce délai est ramené à huit jours ». Journal officiel de la République démocratique du Congo, $52^{\text {ème }}$ année, Numéro Spécial du 5 février 2011.

50 Article 161 al. 3 de la Constitution : «Elle peut en outre, saisir la Cour constitutionnelle par la procédure de l'exception de l'inconstitutionnalité invoquée dans une affaire qui la concerne devant une juridiction ». Op. Cit.

51 Article 93 de loi organique $\mathrm{n}^{\circ}$ 13/026 du 15 octobre 2013 portant organisation et fonctionnement de la cour constitutionnelle qui dispose : « ils ne sont susceptibles d'aucun recours, sauf interprétation ou rectification d'erreur matérielle ». Journal officiel, numéro spécial, $54^{\text {ème }}$ année, 18 octobre 2013.

52 Voy. Articles 100 à 107 de loi organique $\mathrm{n}^{\circ}$ 13/026 du 15 octobre 2013 portant organisation et fonctionnement de la cour constitutionnelle, $\mathrm{Op}$. Cit.

53 Article 161 al. 4 de la Constitution de la RDC qui dispose : « Elle connaît des recours contre les arrêts rendus par la Cour de cassation et le Conseil d'Etat, uniquement en tant qu'ils se prononcent sur l'attribution du litige aux juridictions de l'ordre judiciaire ou administratif. Ce recours n'est recevable que si un déclinatoire de juridiction a été soulevé par ou devant la Cour de cassation ou le Conseil d'Etat ». Op. Cit. 
tances judiciaires ne sont pas des juridictions de jugement. En effet, elles ne statuent pas en principe sur le fond de l'affaire. De ce fait, la Cour militaire opérationnelle reste donc la seule juridiction qui tranche sur la culpabilité (à partir de l'examen des faits) sans autoriser une quelconque voie de recours de ses décisions.

Dans l'affaire dite Minova les parties civiles ont soulevé l'exception d'inconstitutionnalité prévue devant les juridictions militaires par l'article 76 al. 4 du code judicaire militaire qui dispose que : «Elles (les juridictions militaires) sont incompétentes pour statuer sur la constitutionnalité des lois et des actes ayant force de loi. Les exceptions soulevées à cet effet sont portées devant la Cour Suprême de Justice qui statue, toutes affaires cessantes, en tant que Cour Constitutionnelle ». Dans ce cas, la saisine est immédiatement faite par la juridiction concernée en vertu de l'article 162 al.4. Cet article ${ }^{54}$ prévoit la surséance de la juridiction devant laquelle l'exception a été soulevée et la saisine par elle de la Cour Constitutionnelle. Dans ce cas précis, il revenait donc à la Cour militaire opérationnelle de saisir la Cour suprême de justice exerçant de manière transitoire les attributs de la Cour constitutionnelle en vertu de l'article 223 de la Constitution ${ }^{55}$. La même compréhension ressort de la loi portant organisation et fonctionnement de la Cour constitutionnelle qui, en conformité avec la Constitution dispose sur une saisine immédiate après la surséance de la juridiction $^{56}$.

Par la lecture combinée de ces articles sus évoqués, La Cour militaire opérationnelle n'est pas compétente à apprécier l'admissibilité de la requête des parties par un jugement avant dire droit ${ }^{57}$. Ce que lui exige la loi, c'est de saisir immédiatement la Cour Constitutionnelle. La Cour militaire opérationnelle dans l'affaire Minova a appliqué dans ce cas

54 Article 162 al.4 de la Constitution : « Celle-ci [la juridiction devant laquelle l'exception d'inconstitutionnalité est soulevée] sursoit à statuer et saisit, toutes affaires cessantes, la Cour constitutionnelle ». Op. Cit.

55 Article 223 de la Constitution : " En attendant l'installation de la Cour constitutionnelle, du Conseil d'Etat et de la Cour de cassation, la Cour suprême de justice exerce les attributions leur dévolues par la présente Constitution ». Op. Cit.

56 Article 52 de loi organique no 13/026 du 15 octobre 2013 portant organisation et fonctionnement de la cour constitutionnelle : " Hormis les traités et accords internationaux, toute personne peut invoquer l'inconstitutionnalité des actes cités à l'article 43 de la présente Loi organique dans une affaire qui la concerne devant une juridiction. Ce droit est reconnu aussi à la juridiction saisie et au Ministère public. Dans ce cas, la juridiction sursoit à statuer et saisit la Cour toutes affaires cessantes ». Op. Cit.

57 La Cour militaire opérationnelle a appliqué l'art. 133. ORDONNANCE-LOI 82-017 du 31 mars 1982 relative à la procédure devant la Cour suprême de justice qui dispose : «Lorsque les parties ou le ministère public soulèvent l'exception d'inconstitutionnalité d'une loi ou d'un acte du président du Mouvement populaire de la révolution, président de la République ayant valeur de la loi invoquée par l'une des parties et applicable au litige dont une juridiction est saisie, celle-ci statue par jugement sur le rejet ou la prise en considération de l'exception. Lorsqu'elle retient l'exception, la juridiction sursoit à statuer sur les demandes pendantes; elle peut toutefois poursuivre toute procédure d'instruction de la cause et prendre les mesures conservatoires nécessaires ». Consulté sur http://www.droitcongolais.info/files/1.17.16.-Ordonnance-loi-du-31-mars-1982-sur-la-procedure-d evant-la-CSJ.pdf accédé dernièrement le 27 octobre 2016. 
l'article 133 de l'ordonnance portant sur la Procédure devant la Cour suprême de Justice ${ }^{58}$ pourtant abrogé par l'article 119 de la loi portant organisation et fonctionnement de la Cour constitutionnelle ${ }^{59}$.

Le juge de la Cour militaire opérationnelle du Nord Kivu, en rejetant la demande des parties civiles, a privé les parties non seulement du droit d'appel mais également du droit à ce que leur cause soit entendue par le juge que la loi leur assigne. Partant du fait qu'aucun recours n'est possible contre les arrêts de la Cour militaire opérationnelle, il s'en suit donc qu'elle ne peut statuer actuellement sans violer la Constitution.

Plus encore, si dans l'arrêt avant dire droit statuant sur l'exception d'inconstitutionnalité, le juge prétend que les parties civiles n'ont pas clairement identifié la norme qu'elles pensaient inconstitutionnelle, sa motivation reste par ailleurs lacunaire. Deux raisons peuvent ainsi nous en convaincre :

Primo, la loi qui interdit les voies de recours devant la Cour militaire opérationnelle est la loi $n^{\circ} 023 / 2002$ du 18 novembre 2002 portant code judiciaire militaire. Il ne pourrait donc pas s'agir, comme s'interroge la Cour, l'Ordonnance $\mathrm{N}^{\circ}$ 08/003 du 09 Janvier 2008 portant implantation d'une cour militaire opérationnelle ou encore la décision portant désignation à titre provisoire des magistrats de la haute cour militaire à cette cour militaire opérationnelle. C'est la loi de 2002 portant code judiciaire militaire qui garantit la légalité aux deux autres textes $^{60}$ cités par le juge. En effet, c'est elle qui crée et pose l'organisation et la compétence de la Cour et consacre l'absence de recours devant celle-ci.

Secundo, l'art 52 al. 2 de la loi portant organisation et fonctionnement de la cour constitutionnelle cité plus haut ${ }^{61}$ donne également le droit au ministère public et à la juridiction saisie du litige de procéder au contrôle de la constitutionnalité des lois par voie d'exception. $\mathrm{Au}$ regard de son caractère d'ordre public, cette exception peut être soulevée donc d'office. Dans son observation, le Ministère public avait été d'avis avec les parties civiles sur la saisine de la Cour Suprême de justice mais sans dire qu'il soulevait cette exception. Quant à la

58 Cet article dispose : « Lorsque les parties ou le Ministère public soulèvent l'exception d'inconstitutionnalité d'une loi ou d'un acte du président du Mouvement populaire de la révolution, président de la République ayant valeur de la loi invoquée par l'une des parties et applicable au litige dont une juridiction est saisie, celle-ci statue par jugement sur le rejet ou la prise en considération de l'exception. Lorsqu'elle retient l'exception, la juridiction sursoit à statuer sur les demandes pendantes; elle peut toutefois poursuivre toute procédure d'instruction de la cause et prendre les mesures conservatoires nécessaires » 31 mars 1982. - ORDONNANCE-LOI 82-017 relative à la procédure devant la Cour suprême de justice. (J.O.Z., n 7,1 er avril 1982, p. 11), Droit civil et judiciaire, Tome 1, Code Larcier, éd. Afrique, 2003. p. 331.

59 Cet article dispose : « Les titres V et VI de l'Ordonnance-loi no 82-017 du 31 mars 1982 relative à la procédure devant la Cour Suprême de Justice sont abrogés ». Op. Cit. p. 24.

60 Le juge a fait référence à l'Ordonnance $\mathrm{N}^{\circ}$ 08/003 du 09 Janvier 2008 portant implantation d'une Cour Militaire Opérationnelle et à la décision portant désignation à titre provisoire des Magistrats de la Haute Cour Militaire à cette Cour Militaire Opérationnelle. Voy. Cour militaire opérationnelle, RP $\mathrm{N}^{\circ} 003$ Auditeur militaire et parties civiles C / NZALE NKUMU NGANDO et consorts, Jugement avant dire droit, Cinquième feuillet.

61 Voy. Note $n^{\circ} 51$ 
cour, elle est restée muette sur un droit qu'elle aurait dû exercer en vertu de l'article 52 al. 2 .

\section{Conclusion}

Somme toute, le droit d'accès au second juge est un droit garanti par le Pacte international relatif aux droits civils et politiques et la Charte africaine des droits de l'homme et des peuples. Il constitue un des droits composants les garanties à un procès équitable. Toutefois, partant de la formulation des articles qui consacrent ce droit au second juge dans ces instruments, il parait indisponible dans le chef de la partie civile dans un procès pénale. Notre question a porté premièrement sur l'étendu et le titulaire de ce droit. Comme le condamné, la partie civile a également droit à ce double degré de juridiction en vertu de la protection par ricochet. En effet, l'exclure conduirait à une inégalité entre les parties au procès faisant ainsi dépendre la saisine des organes de contrôle du seul condamné. Ainsi, la CMO, telle qu'organisé actuellement, reste une juridiction établie en violation du Pacte international relatif aux droits civils et politiques et de la Charte africaine de droits de l'homme et des peuples vis-à-vis des droits aussi bien de l'accusé que de la partie civile.

Deuxièmement, nous avons critiqué la décision du juge se rapportant à l'exception d'inconstitutionnalité dans l'affaire Minova. La CMO, en application d'une norme pourtant abrogé, a rejeté cette exception. Au regard des dispositions en vigueur, le juge n'aurait pas dû apprécier cette demande en inconstitutionnalité. Elle aurait dû tout simplement saisir la Cour suprême de justice exerçant à titre transitoire les compétences de la Cour Constitutionnelle.

Nous en avons conclu que les parties civiles conservent actuellement d'autre choix pour faire valoir leur droit dans cette affaire. Il s'agit soit du comité de droit de l'homme soit de la commission africaine des droits de l'homme et des peuples. Ces deux organes auront alors pour mission de constater le manquement de la RDC du fait de maintenir une législation incompatible avec ces engagements internationaux. Par ailleurs ce dernier organe reste la plus propice à reconnaître les droits des parties civiles dans cette affaire.

$\mathrm{Au}$ côté de cette question se rapportant au double degré de juridiction en vue du respect au droit à un procès équitable, d'autres questions, juridiques ou non, gardent tout leur sens : Pourquoi les autorités judiciaires auraient-elles attribué cette cause à une juridiction dont le fonctionnement et l'organisation sont contraires non seulement à la Constitution mais aussi aux engagements internationaux de la RDC? En effet, deux autres juridictions auraient pu être compétentes pour connaître de ce litige. Dans ce cas il s'agit des cours militaires de Bukavu et de Goma offrant, elles, la possibilité d'appel ${ }^{62}$. Plus encore, ne serions-nous pas devant une juridiction d'exception pourtant proscrite par la Constitution ${ }^{63}$ ? Le mode de for-

62 L'article 85 du code judiciaire militaire dispose que : « Les arrêts rendus par les Cours Militaires au premier degré sont susceptibles d'opposition et d'appel ». Op. Cit.

63 Article 149 al. 5 de la constitution dispose : « Il ne peut être créé des tribunaux extraordinaires ou d'exception sous quelque dénomination que ce soit». Op. Cit. 
mation de sa composition et de désignation de ses animateurs, son implantation par l'exécutif, etc. sont autant de caractéristiques qui devraient faire l'objet de recherches ultérieures.

La Cour militaire opérationnelle du Nord Kivu a évité de donner à la Cour constitutionnelle l'occasion de dire le droit. Ce qui ne saurait tarder à venir au regard de l'activité de cette Cour dans cette zone de la RDC et des critiques plus élaborées des chercheurs aptes à éclairer les justiciables et leurs conseils.

\section{Bibliographie}

Pacte international relatif aux droits civils et politiques. http://www.ohchr.org/FR/ProfessionalInterest/ Pages/CCPR.aspx.

Premier protocole facultatif se rapportant Pacte international relatif aux droits civils et politiques http:// www.ohchr.org/FR/ProfessionalInterest/Pages/OPCCPR1.aspx.

Charte africaine des droits de l'homme et des peuples http://www.achpr.org/fr/instruments/achpr/

Commission africaine des droits de l'homme et des peuples, Résolution sur la Procédure relative au Droit de Recours et à un Procès Equitable. La Commission Africaine des Droits de l'Homme et des Peuples, réunie en sa 11ème Session Ordinaire, à Tunis Tunisie, du 2 au 9 mars 1992. http://w ww.achpr.org/fr/sessions/11th/resolutions/4/.

Commission africaine des droits de l'homme et des peuples, affaire Interights, ASADHO et Maître Odette Disu c/République Démocratique du Congo, Communication 274/03 et 282/03, p.14. http:// www.achpr.org/fr/communications/decisions/?c=67\&a=863.

Commission africaine des droits de l'homme et des peuples, affaire Interights, ASADHO et Maître Odette Disu c/République Démocratique du Congo, Communication 274/03 et 282/03, p.20. http:// www.achpr.org/fr/communications/decisions/?c=67\&a $=863$.

Nations unies, Déclaration des principes fondamentaux de justice relatifs aux victimes de la criminalité et aux victimes d'abus de pouvoir. Adoptée par l'Assemblée générale des Nations unies dans sa Résolution 40/34 du 29 novembre 1985.

Comité des droits de l'homme des Nations unies, affaire Curie contre la Jamaïque, CCPR/C/44/D/ $377 / 1989$.

Comité des droits de l'homme des Nations unies, affaire Jésus TERRON c/ Espagne, CCPR/C/82/D/ 1073/2002, 15 novembre 2004. p. 7.

Comité des droits de l'homme des Nations unies, affaire M. Valichon Aliboev c/ le Tadjikistan, CCPR/C/85/D/985/2001. 16 novembre 2005. p. 8 .

Constitution telle que modifiée par la Loi $\mathrm{n}^{\circ} 11 / 002$ du 20 janvier 2011 portant révision de certains articles de la Constitution de la République Démocratique du Congo du 18 février 2006. Journal officiel de la République démocratique du Congo, $52^{\mathrm{ème}}$ année, Numéro Spécial du 5 février 2011.

Loi organique $n^{\circ} 13 / 026$ du 15 octobre 2013 portant organisation et fonctionnement de la cour constitutionnelle. Journal officiel, numéro spécial, $54^{\text {ème }}$ année, 18 octobre 2013.

Loi n ${ }^{\circ} 023 / 2002$ du 18 novembre 2002 portant code judiciaire militaire. Journal Officiel - Numéro Spécial-20 mars 2003 . 
Ordonnance $\mathrm{n}^{\circ}$ 08/003 du 09 janvier 2008 portant implantation d'une Cour Militaire Opérationnelle. Journal officiel de la République démocratique du Congo $49^{\mathrm{ème}}$ année, $\mathrm{N}^{\circ} 2$ du 15 janvier 2008.

ORDONNANCE-LOI 82-017 du 31 mars 1982 relative à la procédure devant la Cour suprême de justice. Droit civil et judiciaire, Tome 1, Code Larcier, éd. Afrique, 2003.

Décret-loi n 019 du 23 août 1997 portant institution d'une Cour d'ordre militaire. http://www.achpr.or $\mathrm{g} /$ files/sessions/44th/comunications/281.03/achpr44_281_03_fra.pdf

Cour militaire opérationnelle, $R P N^{\circ} 003$ Auditeur militaire et parties civiles C / NZALE NKUMU NGANDO et consorts, Jugement avant dire droit.

Paul TARVENIER, « La protection des droits de l'homme par le Comité des droits de l'homme des Nations Unies. Les communications individuelles »Revue trimestrielle des droits de l'homme (1996). p.6. http://www.rtdh.eu/pdf/19963.pdf.

Alain Didier OLINGA, « Les emprunts normatifs de la commission africaine des droits de l'homme et des peuples aux systèmes européen et interaméricain de garantie des droits de l'homme ». www.rtdh.eu/pdf/2005499.pdf. 